\title{
Poor Economics - Knowledge Economy and The Existing Knowledge Gaps (Higher and Academic Education) In Healthcare; How To Overcome?
}

\author{
Cees Th. Smit Sibinga ${ }^{1}$, Arwa Z. Al-Riyami², Maruff A. Oladejo ${ }^{3}$, Isaac Kajja ${ }^{4}$ \\ ${ }^{1}$ IQM Consulting, University of Groningen, NL \\ ${ }^{2}$ Sultan Qaboos University Hospital, Muscat \\ ${ }^{3}$ University of Lagos, Nigeria \\ ${ }^{4}$ College of Health Sciences, Makerere University, Kampala, Uganda \\ c.sibinga@planet.nl \\ Oman.arwa@squ.edu.com \\ maoladejo@unilag.edu.ng \\ kajja133@gmail.com
}

\begin{abstract}
The 2018 United Nations Development Program (UNDP) presents clearly that the lower the human development indices (HDI), the more significant the decrement in secondary and tertiary education enrolment (higher and academic). For example, tertiary education in the very high-HDI countries shows $72 \%$ enrolment, where enrolment in the medium and low $\mathrm{HDI}$ parts of the world is only $24 \%$ and $8 \%$ respectively. The data illustrate the impressive paucity in education, hence knowledge production. This paucity in education is predicted to result in a weak knowledge economy of available and accessible knowledge. This root cause analysis discloses a significant area of attention to narrow and bridge the existing knowledge gap. Education in these countries has been focused almost exclusively on vocational education of technical skills with limited theoretical attention (knowledge) and rudimentary attention to topics such as governance, human capacity investment, and appropriate application. Education needs the proper environment and climate at primary, secondary and tertiary education levels, resulting in an effective knowledge economy and contributing to advancing progress and improvement of quality. The healthcare system for example needs to be available and accessible to all. This environment can only be created and developed when countries establish national structures, institutional environment, and a competent leadership and management cadre. This depends on the existence of a well-educated and motivated cadre of 'intelligentsia', competent and responsible policymakers and governors, and creating an inviting and inspiring education climate, irrespective of the level of knowledge to be acquired.The paper provides a global situation analysis of qualified and quantified key elements of the knowledge economy, and analysis of the impact of differentials between knowledge economies of the various human development groups on national healthcare structures and patient safety as a final outcome. Additionally, it alludes to methods on how to improve on the identified gaps.
\end{abstract}

Keywords: poor economics, knowledge gap, higher education, academic education, healthcare, patient safety

\section{Introduction}

National development should be measured not only by income per capita, but also by health and education achievements. Imbalances in people's opportunities and choices stem from inequity in income, education, health, access to technology, among others. At a global scale, inequities in income contribute the most to overall inequities, followed by education and life expectancies. For example adults in very high human development countries average 7.5 years more schooling than those in low human development counties. The United Nations Development Plan (UNDP) 2018 statistical update report 'Human Development Indices and Indicators' presents clearly that the lower the human development indices (HDI), the more significant the decrement in secondary and tertiary (higher education, university) education enrolment (UNDP, 2018). For example, tertiary education in the very high-HDI countries shows $72 \%$ enrolment, whereas enrolment in the medium and low HDI parts of the world, is only $24 \%$ and $8 \%$ respectively (Table 1 ).

Table 1: UNDP 2018 Education enrolment ratio for primary, secondary and tertiary education in the four Human Development Index groups.

\begin{tabular}{|l|l|l|l|}
\hline Human Development Groups & \multicolumn{3}{|c|}{ EDUCATION ENROLMENT RATIO } \\
\hline & $\begin{array}{l}\text { Primary } \\
\text { school-age population } \\
(\%)\end{array}$ & $\begin{array}{l}\text { Secondary } \\
\text { school-age } \\
\text { population } \\
(\%)\end{array}$ & $\begin{array}{l}\text { Tertiary } \\
\text { school-age population } \\
(\%)\end{array}$ \\
\hline
\end{tabular}




\begin{tabular}{|l|c|c|l|}
\hline \multicolumn{3}{|c|}{ Human Development Groups } & \multicolumn{3}{|c|}{ EDUCATION ENROLMENT RATIO } \\
\hline Low HDI & 98 & $\mathbf{4 3}$ & $\mathbf{8}$ \\
\hline Medium HDI & 110 & $\mathbf{7 3}$ & $\mathbf{2 4}$ \\
\hline High HDI & 103 & 96 & $\mathbf{5 0}$ \\
\hline Very-High HDI & 102 & 106 & $\mathbf{7 2}$ \\
\hline
\end{tabular}

$\mathrm{HDI}=$ human development index

Most vocational schools and universities (higher and tertiary education) in many developing countries are relatively young and date back to the last part of the colonial era, the second half of the $19^{\text {th }}$ and the first half of the $20^{\text {th }}$ Centuries. The data illustrate the impressive paucity in education, hence knowledge production, predicting to result in a weak knowledge economy of available and accessible knowledge. This analysis discloses a significant area of attention to narrow and bridge the existing knowledge gap. Education in these countries has been focused almost exclusively on vocational teaching and training of technical skills with limited theoretical attention (knowledge), and rudimentary attention to topics such as governance, human capacity investment, and appropriate application.

Education needs the appropriate environment and climate at primary, secondary, and tertiary levels, to achieve an effective knowledge economy, advance progress and improve quality for instance in a healthcare system that is available and accessible to all (WHO, 2021b). This environment can only be created and developed when countries establish national structures, institutional environment, competent leadership and management cadre. This depends on the existence of a well-educated and motivated cadre of 'intelligentsia', competent and responsible policymakers and governors, and creating an inviting and inspiring education climate, irrespective of the level of knowledge to be acquired (Smit Sibinga, et al., 2021) and the economy of using and exchanging the knowledge

Against this background the paper will provide information on key elements and characteristics of knowledge economy, the impact of knowledge economy and the World Bank knowledge economy pillars on the development of the healthcare system, and a section on knowledge economy and the current WHO Patient Safety Action Plan 2021-2030, ending with conclusive remarks and policy recommendations.

\section{Key elements and characteristics of knowledge economy}

Knowledge economy is a system of consumption and production that is based on intellectual capital. It is an economy where knowledge is acquired, created, spread, and used effectively to enhance economic development, whether for profit or not for profit, private or public (Dang and Umemoto, 2009). It is also defined as production of services based on knowledge-intensive activities that contribute to an accelerated pace of technical and scientific advance, as well as rapid obsolescence (Powell and Snellman, 2004).

Successful knowledge economy typically involves modernizing the information infrastructure, long-term investments in education, developing innovation capability, and having an economic environment conducive to market transactions (Chen and Dahlman, 2006). These elements have been advocated as the pillars of the Knowledge Economy. There are four pillars that together constitute the Knowledge Economy framework (Figure 1):

1. An institutional and economic incentive regime that provides good economic policies and education institutions that permit efficient mobilization and allocation of resources, and stimulate creativity and incentives for the efficient creation, dissemination, and use of existing knowledge.

2. Educated (knowledge) and skilled people at any level of education who can continuously upgrade and adapt their skills to create and use knowledge efficiently.

3. An effective innovation system of educational institutions, research centres, universities, consultants, and other organizations that can keep up with the knowledge revolution, and connect to the growing library of global knowledge, assimilate and adapt it to local needs e.g., healthcare.

4. A modern and adequate information infrastructure that can facilitate the effective communication, dissemination, and processing of information and knowledge e.g., the development of operational artificial intelligence [Al - artificial neural networks (ANN), deep learning (DL) and machine learning $(\mathrm{ML})]$. 


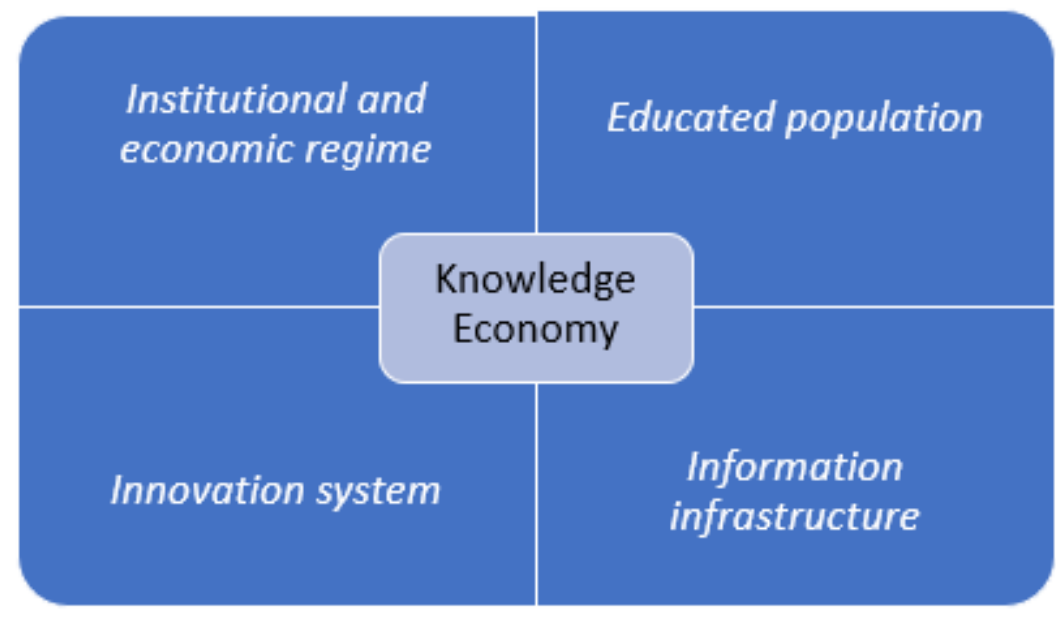

Figure 1: Framework of knowledge economy

Such framework asserts that investment in the four knowledge economy pillars is necessary for sustained creation, adoption, adaptation, and use of knowledge in domestic economic processes, resulting in higher valueadded outcomes, goods, and services as exemplary in the healthcare.

\subsection{Human Capital}

Knowledge economy is focused on the importance of human capital in the economy of the $21^{\text {st }}$ Century through assets, relations and capability views (Dang and Umemoto, 2009). The rapid expansion of knowledge and the increasing reliance on computerization, big data analytics, robotics and automation is changing the economy of the advanced world to one that is even more dependent on intellectual capital and managerial skills, and less dependent on the technical production processes. This unfortunately, results in increasing the existing knowledge gaps in different parts of the world, due to its emphasis on specific knowledge and skills, data analysis and measurable performance, and strategic management by objectives.

Knowledge economy is characterized by the presence of a higher percentage of highly skilled employees whose jobs require advanced and special knowledge and skills. In the past knowledge economy depended heavily on low- and unskilled labour jobs in industry and agriculture and consisted primarily of producing physical goods, the modern economy is comprised more of services and jobs that require intellectual capacities and analysis of data produced by robotics and computerized processes and procedures (machine learning). This results in an increased demand for highly skilled and educated labour relative to the demand for low-skilled labour, suggesting that technological change contributes more to the productivity of highly educated workers (Powell and Snellman, 2004).

The modern economy - also known as the post-industrial economy or the information economy - is a reference to the importance of information and communication technology (ICT) and Al in the economies of the world, and an important platform for applied research and science to build on evidence (Chen and Dahlman, 2006). Knowledge economy supports, and is fuelled by innovation, research, and rapid technological advancements such as Al and mega-capacity quantum computers.

\subsection{Intangible Assets}

In the 'new' knowledge economy, the most valuable assets owned by an organization or institution are often intangible assets, such as service, experience, commitment and awareness, or tangible such as proprietary software or processes. This contrasts with previous economic epochs of the $19^{\text {th }}$ and $20^{\text {th }}$ Centuries - for instance the agrarian economy, where the primary asset usually was land, and the industrial economy, where mechanical manufacturing plants and equipment were crucial assets for most industries. The majority of the younger generation working in the current knowledge economy settings is incredibly computer literate and skilled at handling and manipulating data as well as creating data monitoring and evaluation models. Noticeable is an increasing emphasis on quality data collection and analysis, and the development of algorithms and deep learning (multiple hidden layers of artificial neural networks), and machine learning principles. 
The knowledge economy is considered the primary driver of the massive expansion of what is known as 'science, technology, engineering, and mathematics' (STEM) jobs (Giffi et al, 2015). Careers within the STEM fields - which includes occupational paths such as computer science, engineering, chemistry, biology, and medicine - belong to the greatest opportunities for career advancement, higher compensation, and top-level executive positions (STEM Learning). These STEM fields show a rapid expansion in operational size, allowing a high degree of standardization, quality management, and economic efficacy. However, the bottom line is that knowledge provides the foundation for the necessary technical expertise, data collection and analysis skills, and innovative management practices that enable non-profit organizations and institutions such as education and healthcare institutions to compete in the modern global economy. In addition specialized knowledge and skills may serve as either productive assets to deploy, or as products to offer and market on a cost-recovery principle, e.g., in the healthcare.

Another characteristic element of knowledge economy is consolidation of essential processes. An example would be the Blood Supply where mandatory safety testing and processing of collected human blood of smaller non-viable blood banks into larger economies of scale, centred in a strategic geographic area and affiliated to a university or one or more sizable tertiary healthcare institutions, clustering available knowledge and skills, offering better effectiveness and balanced knowledge economy (WHO, 2021a).

Today knowledge economy has become the primary economy among advanced countries, is an economy dependent on human capital and intangible assets, such as service and trust, or proprietary technology. Knowledge economy has placed the ICT and Al sciences and industries at the forefront of overall economic growth through larger and faster computing capacities. Skills sets that include data analysis, creating and working with big data and cost-recovery financial models, and the ability to innovate, based on well-structured higher and academic vocational and professional education systems, are a priority in the modern economy. A change in knowledge and skills requirements and a shift in assets through a different approach in customer orientation and appreciation, both with the community as well as for instance with the healthcare organizations and institutes add on the one side another valuable dimension to quality culture. However, the downside is a widening of the existing knowledge gaps in the less advanced world.

\section{Impact of knowledge economy on the development of the Healthcare system}

Knowledge is gained and acquired through education, learning and experience. Knowledge is offered from the micro and macro environment as information to be received, stored, perceived, interpreted, and converted into signals for action - recognition, behaviour, expression, understanding, thinking, etc. This chain of events starts with birth and builds up during life. To standardize a basic amount of knowledge, teaching and learning systems, processes and curricula have been created and are continuously further developed; contextually, operationally, and technically through e-learning concepts (Kaplan, 2021). Depending on an individual's intelligence and IQ level, education could be adapted and adjusted to what maximally can be perceived and understood or interpreted. The more exposure and repetition, the better the perception, interpretation, understanding, and storage achieved. For each of the three successive layers of structured education, primary, secondary, and tertiary, there is a continuous education and stimulation system to improve and expand on existing knowledge, optimizing the economy of knowledge.

As explained in the introduction, education, and the school-population progression rates from primary into secondary, and secondary into tertiary education are utilized as human development index indicators used by the UNDP (UNDP, 2018). These illustrate the growing quantitative knowledge economy gaps between education levels in the HDI groups. However, more importantly is the quality and depth of knowledge offered and perceived. Education is neither limited nor restricted to what structurally is offered. Mankind is continuously exposed to environmental information in its different forms, visual and pictorial (objects, light and darkness, colours, movement, postures, reading), audible (sound and music, soft and loud, harmonious and dissonant, attracting and warning), touchable (size, shape, surface, smooth and sharp), smell and taste (sweet, bitter, salt, rotten and challenging), senses like temperatures and pain, balance and speed; most come in combinations and environmental dimensions. The personal perception, interpretation, storage, and triggers to action are part of the individual knowledge economy with its levels of alertness and awareness, comfort and discomfort, risks, and safety perceptions (Thomas and Thomas, 1928). The intensity of the experience is part of a common life knowledge economy. 


\subsection{Added Value}

The 2018 UNDP statistical data reveal remarkable differences in acquiring a structured knowledge economy and consequently the value added to education in different parts of the world (UNDP, 2018). This implies that the economy of acquired knowledge is very fundamental and relates closely to the need to survive because of poor economics. The lower the HDI, the smaller the intelligentsia cadre and opportunities to offer and guarantee quality education to a broader community. As a result, the lower the HDI, the more significant the decrement in enrolment rates into secondary and even more prominently into tertiary education. On the more specific healthcare scale, the availability of expertise and/or training programmes in the low and medium HDI countries is much more restricted than in the higher HDI countries (Smit Sibinga et al., 2021). Currently, advanced technology allows us to bridge this gap to an extent by having blended teaching and online learning and teaching programmes, ideally developed, and presented jointly by local and international experts, at relatively low cost with no great need for travel.

This analysis illustrates the need for a specific knowledge pool to be deployed economically. Personnel with a secondary education background are exposed to continued education at a higher and academic (tertiary) education level to graduate as a vocational professional. These vocational professionals then form the pool of (graduate) trainees for an operational or managerial position. As an example, an optimal and quality knowledge economy can help to achieve the ultimate goal in the healthcare of improving patient safety, preventing avoidable harm and achieving better health outcomes in different healthcare systems (WHO, 2021b). Unfortunately, the pool of healthcare graduates is limited in medium and low HDI countries (enrolment ratios $24 \%$ and $8 \%$ resp.) and marginal (enrolment ratio 50\%) in high HDI countries (Table 1) (UNDP, 2018). That brings along a serious competition for a job at that professional level in the healthcare institutions and hospitals, and the risk for a drain of well-equipped professionals away from the smaller institutes to larger hospitals with larger and more diverse and occasionally more advanced diagnostic and treatment programmes.

\subsection{Operational Knowledge in the healthcare system}

An analysis of operational knowledge and skills in the healthcare (primary and in hospitals) illustrates that the educational or knowledge acquisition focus and emphasis points to three integrated groups or categories of health professionals to be educated - doctors, nurses, and (bio-)medical or chemical laboratory professionals, supported by ancillary personnel who do not need a specific healthcare education and knowledge. Most ancillary personnel require a primary or secondary education background and may receive structured apprentice or workintegrated education and training related to professional behaviour (professionalism), institutional values, infection control, safety and risk prevention, in line with their respective roles and responsibilities (Smit Sibinga et al, 2021). Knowledge on professionalism and stewardship is essential at the early stage of entry and training, appropriate to the role and function of the various ancillary personnel (Rambiritch and Smith-Tolken, 2019).

Unsafe medical practices and unnecessary interventions expose patients to the risk of serious adverse events and cause unnecessary harm. Unnecessary interventions also reduce the availability of care and attention for patients who are in need (WHO, 2021b). Therefore, it is recommended to develop systems and benchmarking tools, such as patient blood management (PBM), haemovigilance, and pharmacovigilance, to monitor and improve the safety of clinical treatment processes (Murphy, Saxena and Smit Sibinga, 2013) and outcomes.

\section{World Bank knowledge economy pillars and the Healthcare system}

When benchmarking development in healthcare systems against the mentioned four World Bank knowledge economy pillars (Figure 1) (Chen and Dahlman, 2006), the following could be observed and implemented:

\subsection{Ad 1 - Institutional structures that provide incentives for entrepreneurship and the active use of knowledge -}

The institutional and economic regime like healthcare and integrated support systems like the blood supply need to be such that governance and leadership have incentives for the creation and efficient utilization of knowledge, and thus should have well-grounded and transparent macroeconomic, competition, and regulatory policies. A 'knowledge-conducive' regime, in general, will be free from various protectionist policies in order to foster development through healthy competition, which in turn will encourage entrepreneurship. Government expenditures and budget deficits should be containable, and inflation should be stable and low. The exchange rate should be stable and reflect the true value of the currency to allow uninterrupted imported supplies of 
consumables, diagnostics, reagents, test kits, and equipment. The financial system should preferably be one based on cost-recovery, able to allocate resources to sound investment opportunities, and redeploy assets from failed enterprises to more promising ones through consolidation and creation of effective operational economies of scale.

\subsection{Ad 2 - Availability of competent (knowledge and skills) labour and a good quality education system -}

A well-educated and skilled population is essential to the efficient creation, acquisition, dissemination, and utilization of relevant knowledge, increasing total productivity and hence economic growth and preventing shortages. Basic or primary education is necessary to increase the capacity of learning and to use information. On the other hand, technical secondary-level education, and higher education in engineering and scientific areas is necessary for technological innovation and adaptation of foreign technologies for use in domestic production processes such as the manufacture of pharmaceuticals or blood products. The production of new knowledge and its adaptation to a particular economic setting is generally associated with higher-level teaching and research. Such training is necessary to monitor technological trends, assess what is relevant for the healthcare, and assimilate new technologies. A more educated population also tends to be relatively more technologically sophisticated and competent. This generates a local quality-sensitive demand for advanced medicinal products, which in turn tends to stimulate hospitals to innovate and design technologically sophisticated interventions and techniques such as use of extracorporeal membrane oxygenation (ECMO) in neonatology (Luban and Short, 2005) and cellular therapies in haemato-oncology (Rebulla, Pacchiana, Montemurro, et al.2003).

\subsection{Ad 3 - Access to and exchange of information and communication technology (ICT and Al) infrastructures -}

Information and communication technologies (ICTS) and Al infrastructure in an economy refers to the accessibility, reliability, capacities, efficiency and speed of computers, smart phones, television and radio sets, and the various networks that link them. ICTs consist of hardware, software, networks, and media for the collection, storage, processing, transmission, and presentation of information in the form of voice, data, text, and images. Apart from increasing the supply of information and knowledge, ICTs can overcome geographic boundaries.

ICTs are the backbone of the knowledge economy. and have been recognized to be effective for promoting economic growth and sustainable development. With relatively low usage costs and the ability to overcome distance, ICTs have revolutionized the transfer of information and knowledge worldwide. The past decade have shown that ICT production and ICT usage have contributed to economic or operational growth. One of the most obvious benefits associated with ICT usage is the increased flow of information and knowledge. Because ICTs allow information to be transmitted relatively inexpensively and efficiently (in terms of cost), ICT usage tends to reduce uncertainty and transactions costs of participating in transactions. This tends to lead to an increase in the volume of transactions leading to a higher level of output and productivity. For instance, in the healthcare system, technologies have been acquired and adapted to increased innovation and productivity, reduction in errors, unwanted deviations from standards, and mitigation of patient harm.

\subsection{Ad 4 - A vibrant innovation landscape that includes academia, the private and government sectors, and civil society -}

Theoretical economy indicates that technological progress is a significant source of productivity growth, and an effective innovation system is key for such technical advancement whether industrial or in healthcare. An innovation system refers to the network of institutions, rules, and procedures that influence how a country acquires, creates, disseminates, and uses knowledge - the knowledge economy. Institutions in the innovation system include universities, healthcare institutions, public and private research centers and policy think tanks. Non-governmental organizations and the government are also part of the innovation system to the extent that they also produce new knowledge.

An effective innovation system provides an environment and climate that nurtures research and development (R\&D), which results in new products and services, new processes and new knowledge and experience, and hence is a major source of technical progress and development. 
Currently, most technical knowledge is produced in advanced countries: more than $70 \%$ of production of scientific and technical publications are accredited to researchers in advanced countries (Hayes, 2004). The disparity in the production of technical knowledge per capita between advanced and developing countries is even more significant than the income disparity. However, domestic technological innovation is not the sole source of the generation of technical knowledge. There are many ways for developing countries to avoid reinventing the wheel and tap into, adopt, and adapt technical knowledge created in advanced countries. A vital element of an innovation strategy of a developing country is to find the best ways to tap into the growing global knowledge-base and decide where and how to deploy its domestic R\&D capability to strengthen existing education systems and research and development structures and accelerate the pace of development of the many weak healthcare systems and research institutes. These are of paramount importance for a successful implementation of the UN (UN, 2015) and WHO (WHO, 2019, 2020) initiatives and development programmes on poverty, education, healthcare and patient safety, and environment.

\section{Knowledge economy and the WHO Global Patient Safety Action Plan 2021-2030}

Over the next decade, much work is to be done to accomplish the set goals of the 2012 Universal Health Coverage (UHC) programme (WHO, 2012), an inspiring goal whereby all individuals and communities receive safe and quality health services without suffering financial hardship by 2030.

This target can be achieved if the world stays on track to achieve the 2016-2030 Sustainable Development Goals (SDGs) (UN, 2015) and the numerous goals in the WHO Thirteenth General Programme of Work (WHO, 2019). However, the SDG agenda will not be met without ensuring that health services are safe. In the absence of such assurance, the benefits of increased coverage cannot be fully realized, and people may experience reduced trust in health services and reduced willingness to seek healthcare - even when they most need it, leading to a steady rise in avoidable harm. May 2019 the $72^{\text {nd }}$ World Health Assembly adopted resolution WHA72.6 on "Global action on patient safety" to give priority to patient safety as an essential and fundamental step in building, designing, operating, and evaluating the performance of all healthcare systems (WHA, 2019). Adopting of this resolution was a remarkable milestone in global efforts to take concerted action on patient safety and reduce the burden of patient harm due to unsafe health care, based on an improved knowledge economy. To move forward from global commitment to tangible action, WHO launched a flagship initiative in February 2020: The Decade of Patient Safety 2020-2030 (WHO, 2020). The challenge for all health systems and all organizations providing health care is to maintain a heightened awareness to detect safety risks and address all sources of potential harm to which accurate data collection is paramount. Patient safety is a framework of organized activities that creates cultures, processes, procedures, behaviours, technologies and environments in healthcare that consistently and sustainably lower risks, reduce the occurrence of avoidable harm, make error less likely and reduce the impact of harm when it does occur. The practice of patient safety involves coordinated action to prevent harm to patients, caused by the processes of health care themselves. Patient safety is a strategic priority for modern healthcare and is central to efforts in the country working towards universal health coverage. The better healthcare and risk prevention knowledge are applied, the safer the offered care will be for the patient community in a country.

Currently, the burden of injuries and other harm to patients from adverse events during medical interventions and nursing is likely one of the top 10 causes of death and disability globally, comparable to that of tuberculosis and malaria. Available evidence suggests that most of this burden falls on low- and middle-income countries, where annually in hospitals, around 134 million healthcare-associated adverse events occur due to unsafe care and contributing to some 2.6 million deaths (WHO, 2021b).

\subsection{Strategic Objectives}

The initiation of the Global Patient Safety Action Plan 2021-2030 (WHO, 2021b) was endorsed during the WHA in May 2021 and focuses on eliminating avoidable harm in health care. The Plan has 7 Strategic Objectives and 35 actions to be implemented step by step over the decade. Looking at the Strategic Objectives it becomes clear that a key element is the way knowledge is to be acquired and applied in an economic way to implement and achieve the objectives and, more importantly to sustain the outcomes through stewardship of the stakeholders involved. The principal partners in action are the governments and the existing healthcare facilities and services. However, even more important is the parallel development of the education infrastructure and environment to facilitate quality education at all levels based on expected outcomes, needed to educate an intellectual workforce to - 
1. do zero avoidable harm to patients, a state of mind and a rule of engagement in the planning and delivery of health care all over the world.

2. build high-reliability health systems and health organizations that protect patients daily from harm.

3. to assure the safety of every clinical process.

4. engage and empower patients and families to help and support the journey to safer health care.

5. inspire, educate, skill, and protect health workers from contributing to the design and delivery of safe care systems.

6. ensure a constant flow of information and knowledge to drive the mitigation of risk, a reduction in levels of avoidable harm, and improvements in the safety of care.

7. develop and sustain multisectoral and multinational synergies, partnership, and solidarity to improve patient safety and quality of care.

\subsection{A Broader Approach}

A much broader approach to patient safety is required for countries with limited resources receiving visits from clinical experts from more affluent countries. What is needed from visitors to a hospital in a low-income country is someone who knows how to establish a safe clinical waste facility, or teach the maintenance of a neonatal incubator, or address the nutritional status of patients. This broader thinking on what constitutes safe clinical care is also required in countries where there is conflict and political instability. There will already be a lack of resources, but the presence of weakened healthcare systems and structures will have greatly increased the need for health care. Large cross-border refugee or migrating populations and encampments, as well as poor hygiene and sanitation contributing to frequent disease epidemics, create enormous challenges. The humanitarian agencies have much wisdom and experience to contribute.

These examples highlight the need for a structured and systems approach in the design of clinical processes. The requirements for safe design will vary from country to country depending on the circumstances and situation, so processes must be tailored accordingly - economics, culture, infrastructure, governance, geographics, climate and distances and above all a balanced knowledge economy. There is no one single approach for all settings.

Although all health workers are committed to keeping their patients safe, the majority will believe that they are discharging this commitment through practising within the ethical code of practice, which is synonymous with being a member of their profession. Fewer will think beyond this to fully appreciate the scope of the risks involved in the delivery of healthcare and the scale of avoidable harm, including preventable and treatable harm, that arise daily within every healthcare system in the world. This lack of awareness and understanding of such an important problem amongst many providers at the point of care may seem puzzling. However, it is certainly not because of any lack of compassion on the part of healthcare professionals.

It is because traditional undergraduate, postgraduate, and continuing education programmes place emphasis on evidence-based practice and standards that are disease or clinical condition oriented. The systems aspects of safety issues are often missing, and programmes provide no training on human factors hence a gap in the provision of knowledge and its economy. It is essential that all health workers, managers and leaders understand patient safety. They must be clear about the nature and importance of risk and how harm is generated, the core concepts of patient safety knowledge and science, the ways in which the causes of unsafe care are investigated and understood, and the actions necessary to ensure that care, and its constituent individual processes, is as safe as is possible - a true managing of knowledge economy.

\subsection{Patient Safety Education and knowledge production}

WHO has published the Patient safety curriculum guide for medical schools, complemented by a multiprofessional edition (WHO, 2011). Both have been widely disseminated but have been adopted only in some countries. Major groups of health care providers around the world have developed patient safety educational curricula, and regulatory and professional education bodies in different countries.

Despite this, the influence of these initiatives on existing curricula has been minimal. The challenge is not in creating policies, it is in their implementation. There are multiple barriers to ensuring that patient safety is a significant component of education and training programmes. These include lack of curriculum space, absence of buy-in from stakeholders, weaknesses in educational coordination and planning, limited leadership interest, and insufficient senior medical and nursing champions. These disclose a failing knowledge economy. Several factors have impeded patient safety education, including: 
- unfamiliarity of educators or trainers with teaching patient safety as a new area of knowledge and learning.

- reluctance by academic institutions to teach knowledge outside clinical disciplines to health care students because of existing complete curricula.

- $\quad$ failure of education to keep pace with technological and system advances for safe care.

In many low- and medium-income settings, there is not even sufficient training within a discipline. For example, radiation therapists might practice in their specialty without being in any formal, accredited training programme. It then becomes even more challenging to train them in patient safety without basic training in their specialty, illustrating another aspect of failing knowledge economy. Traditionally, education of healthcare professionals give little attention to the importance of patient safety, as a consequence of which

- there is no professional ethos that a practitioner's responsibilities must extend beyond the care of individual patients to ensuring that their service as a whole is safe.

- there is little understanding of the nature of risk in health care and the importance of strengthening systems.

- there is minimal emphasis on the importance of teamwork and communication in protecting patients from harm.

- there is no understanding of the value of an appropriate and efficacious knowledge economy.

Looking at best practices within healthcare and other high-risk industries, it is clear that new radical approaches, including interprofessional and multidisciplinary approaches, are needed if education (knowledge) and training (skills) are to play the full role that they should in improving patient safety surrounded by adequate knowledge economy valuing a countries investment in education.

These observations are not restricted to health care but can be seen in many other fields within a country's socio-economic operations.

\section{Conclusions and policy recommendations}

In summary, knowledge economy is dependent on human capital and intangible assets, such as service and trust or proprietary technology, hence became primary in developed countries. The change in knowledge and skills requirements and a shift in assets through a different approach in customer orientation and appreciation, both with the community as well as with the healthcare organizations include data collection and analysis, creating and working with big data, and cost-recovery financial models, and the ability to innovate, based on wellstructured higher and academic vocational and professional education systems. It has placed ICT and Al science and industries at the forefront of overall economic growth through larger and faster computing capacities.

Knowledge economy adds on the one side another valuable dimension to quality culture. However, the downside is a widening of the existing gaps. From the analysis the most essential key elements of knowledge economy to overcome the existing higher and academic education gaps are in the formulation of workable policies that will ensure and guarantee-

- a well-constructed and sustainable education environment, climate and adaptive curricular structure (students, teachers and institutional governance).

- a well-balanced and designed education scope and quality of knowledge.

- intellectual property, stewardship, and ownership.

These elements need a high, sustained, and devoted prioritization in the development to which economic spending, sharing, and exchanging knowledge is paramount. These should be supported and accompanied by a genuine curiosity, continued exploration and a healthy appetite to science and evidence-based knowledge that drive development and determine the pace of acceleration towards advancement and improved effectiveness - safety, availability, accessibility, affordability, equity and equality (Crouch, Rolleston and Gustafsson, 2020), . The available and expanding knowledge should actively and purposeful be shared and exchanged through integrity-based collaboration and cooperation. The quantitative statistical data presented (UNDP, 2018) disclose the enormous differences in UNDP indices and indicators state of development brought about by weak and brittle governance, regulations, leadership and stewardship structures and cultures in an impressively large part and population in the world. This can be solved through the awareness and understanding of those who have the knowledge, willing to share and exchange, and convinced that such attitude is the missing key needed to 
unlock and access knowledge as a common human intellectual property to development and progress improvement.

\section{References}

Chen, D.H.C. and Dahlman, C.J. 2006. The Knowledge Economy, the KAM Methodology and World Bank Operations. World Bank Institute, Washington, DC. Stock No. 37258

Crouch L, Rolleston C. and Gustafsson M. 2020. Eliminating global learning poverty: The importance of equalities and equity. International Journal of Education Development; DOI 10.1016/j.ijedudev.2020.102250, on line

Dang D. and Umemoto K. 2009. Modeling the development towards knowledge economy: a national capability approach. Journal of Knowledge Management 13:359-372

Giffi, G., Dollar, B., Gangula, B. and Rodriguez, D. 2015. Help Wanted. American manufacturing competitiveness and the looming skills gap. Deloitte Review 16: 97-113

Hayes, H. 2004, The role of libraries in the knowledge economy. Serials;17:231-238

Kaplan A. 2021. Higher Education at the Crossroads of Disruption: The University of the 21st Century, Great Debates in Higher Education. Emerald Publishing, UK.

Luban N.L.C. and Short B.L. Extracorporeal Membrane Oxygenation on the neonate with respiratory failure. 2005. In: Smit Sibinga C.Th. and Luban N.L.C. eds. Neonatology and Blood Transfusion. Springer, Dordrecht, Netherlands Pp. 217230

Murphy M.E., Saxena S. and Smit Sibinga C.Th. 2013. Safety and Quality Management at the Clinical Interface. In: Smit Sibinga C. Th. ed. Quality Management in Transfusion Medicine. Nova Science Publ, New York. Pp 283-314.

Powell W.W. and Snellman K. 2004. The knowledge economy. Annual Review of Sociology 30:199-220

Rambiritch, V. and Smith-Tolken, A. 2019. The imperative of teaching professionalism to biomedical technologists. African Journal of Health Professionals Education 11:139-144.

Rebulla P., Pacchiana R., Montemurro T., et al. 2003. New horizons in Cellular Therapies. In: Smit Sibinga C.Th. and de Leij L.E.M.H., eds. Cellular Engineering and Cellular Therapies. Kluwer Academic Publ, Dordrecht, Netherlands. Pp. 189201

Smit Sibinga, C.Th., Louw, V.J., Nedelcu, E., Al-Riyami, A.Z., Bakhtary, S., Johnson, S.T., Rambiritch, V., Seoraj, V., Callum, J., Van den Berg, K., Vermeulen, M., Barrett, C.A., \& Eichbaum, Q.G. 2021. Modeling Global Transfusion Medicine Education. Transfusion;61:3040-3049, DOI 10.1111/trf.16641 on line

STEM. 2020. https://www.stem.org.uk/stem-careers [Accessed November 13, 2021]

Thomas, W.I. \& Thomas, D.S. 1928. The Child in America. Alfred Knopf. New York

UN 2015. Sustainable Development Goals 2016-2030. https://sustainabledevelopment.un.org/?menu=1300 [Accessed August 29, 2021]

UNDP 2018. 2018 Statistical Update: Human Development Indices and Indicators. New York. http://hdr.undp.org/en/content/human-development-indices-indicators-2018-statistical-update [Accessed August 29, 2021]

WHA Resolution 72.6 2019. Global action on patient safety. https://apps.who.int/gb/ebwha/pdf files/WHA72/A72 R6en.pdf [Accessed August 29, 2021]

WHO 2011. Patient safety curriculum guide for medical schools. https://www.ihffih.org/download doc file.php?doc=7d49137171e83b8d6906ac1812af1d8b [Accessed August 29, 2021]

WHO 2012. Universal Health Coverage Program. http://www.who.int/universal health coverage/en/[Accessed August 29, 2021]

WHO 2019. Thirteenth General Programme of Work 2019-2023. pdf available at https://sustainabledevelopment.un.org/?menu=1300

WHO 2020. The Decade of Patient Safety 2020-2030. http://www.ihffih.org/download doc file.php?doc=7d49137171e83b8d6906ac1812af1d8b [Accessed August 29, 2021]

WHO 2021a. Guidance on Centralization of Blood Donation Testing and Processing. Geneva, CH. Licence: CC BY-NC-SDA 3.0 IGO

WHO 2021b. Global Patient Safety Action Plan 2021-2030. Towards eliminating avoidable harm in health care. https://www.who.int/docs/default-source/patient-safety/global-patient-safety-action-plan-2021-2030 thirddraft january-2021 web.pdf?sfvrsn=948f15d5 17 [Accessed August 29, 2021] 